\title{
Density fluctuations near the liquid-gas critical point of a confined fluid
}

\author{
Y. B. Melnichenko and G. D. Wignall \\ Condensed Matter Sciences Division, Oak Ridge National Laboratory, Oak Ridge, Tennessee 37831-6393, USA \\ D. R. Cole \\ Chemical Sciences Division, Oak Ridge National Laboratory, Oak Ridge, Tennessee 37831-6110, USA \\ H. Frielinghaus \\ Forschunszentrum Jülich GmbH, Institut für Festkörperforschung, D-52425 Jülich, Germany
}

(Received 27 August 2003; published 24 May 2004)

\begin{abstract}
We report the results of an experimental study of the effect of a dilute silica network on liquid-gas critical phenomena in carbon dioxide $\left(\mathrm{CO}_{2}\right)$. Using small-angle neutron scattering, we measured the correlation length of the density fluctuations in bulk $\left(\xi_{\text {bulk }}\right)$ and confined $\mathrm{CO}_{2}\left(\xi_{\text {conf }}\right)$ as a function of temperature and average fluid density. We find that quenched disorder induced by an aerogel suppresses density fluctuations: $\xi_{\text {conf }}$ loses the Ising model divergence characteristic of $\xi_{\text {bulk }}$ and does not exceed the size of pores in the homogeneous region.
\end{abstract}

DOI: 10.1103/PhysRevE.69.057102

PACS number(s): 64.60.Fr, 64.70.Fx, 68.03.Fg

The behavior of fluids in disordered porous materials has been the subject of intense experimental and theoretical interest over the last decade [1]. A detailed assessment of the role of disorder, confinement, and interfacial phenomena is required in order to quantify the behavior of such systems. The most popular theoretical approach for interpretation of the critical behavior of fluids absorbed in porous media such as Vycor glass or aerogels is the random field Ising model (RFIM) [2]. de Gennes proposed that the porous medium can act as the "random" field because of its preference for one of the binary liquids over the other or its preference for the liquid phase over the vapor phase [3]. Considering the problem from a different perspective, Liu et al. [4] formulated the "single-pore" or "wetting" model which predicts the formation of frozen microdomains below the critical temperature $T_{C}$ in each individual pore independently of randomness. According to this model, the behavior of binary solutions in small pores is defined by the molecule-surface interactions, which reduce the rate of domain growth near $T_{C}$ and thus inhibit macroscopic phase separation. Experimental studies of liquid-liquid phase transitions under confinement have revealed some features consistent with the RFIM, such as the absence of macroscopic phase separation, slow kinetics, and the existence of long-lived metastable states in the two-phase region [5]. Conversely, it was argued [6] that many of the observations can be equally well rationalized in terms of the single-pore model.

As described in the literature, the RFIM should be relevant only at temperatures where the correlation length of the order parameter fluctuations, $\xi$, is larger than the length scale of the random field (i.e., of the order of the pore radius $R_{P}$ ). The most important argument in favor of the single-pore model has been the applicability of the RFIM to liquid-liquid critical phenomena in pores based on the observation that liquid-liquid phase demixing always occurs before $\xi$ exceeds $R_{P}$ [6-8]. Three major factors-i.e., the experimental method, porous matrix, and confined system-should be carefully chosen in order to satisfy the limit $\xi \gg R_{P}$. Small- angle neutron scattering (SANS) is ideally suited for exploring the behavior of fluids in small pores as it can probe the fluctuations of the order parameter (i.e., concentration in solutions or density in fluids) in the pertinent range $\sim 5 \leqslant \xi$ $\leqslant \sim 1000 \AA$ [9]. Aerogels represent a potentially suitable host matrix of highly dilute porous glasses characterized by open pore structure composed of thin interconnected silica strands [10], which can act as quenched dilute impurities embedded in a homogeneous fluid. Investigation of individual fluids rather than multicomponent solutions may be advantageous in order to avoid complications caused by the local composition gradients in pores [6,7]. In addition, onephase fluid systems circumvent the problem of extremely sluggish kinetics in the liquid-liquid critical region [11] which is controlled by mutual diffusion rather than orders of magnitude faster hydrodynamic equilibration. In this Brief Report we report the results of the first SANS measurements of the correlation length of density fluctuations in carbon dioxide $\left(\mathrm{CO}_{2}\right)$ confined in silica aerogel near its liquid-gas critical point. Although we expect $\xi$ of the confined $\mathrm{CO}_{2}$ to grow freely and finally cross over from the $\xi \ll R_{P}$ to $\xi$ $\gg R_{P}$ regimes as $T \Rightarrow T_{C}$, we found unexpected evidence of complete suppression of the density fluctuations in the critical region and formation of the frozen metastable microdomains below $T_{C}$.

A silica aerogel with $\sim 96 \%$ porosity, a surface area of $400 \mathrm{~m}^{2} / \mathrm{g}$, a density of $0.1 \mathrm{~g} / \mathrm{cm}^{3}$, and pore size distribution peaked around the nominal pore diameters $2 R_{P} \sim 60-70 \AA$ [12] was obtained from Oscellus Technologies, Livermore, $\mathrm{CA}$. The aerogel surface is hydrophobic due to the presence of abundant methoxy groups $\left(=\mathrm{Si}-\mathrm{O}-\mathrm{CH}_{3}\right)$ formed during supercritical drying of the precursor gel in supercritical methanol [12]. Aerogel was shaped into a cylinder $(17 \mathrm{~mm}$ o.d., $10 \mathrm{~mm}$ length) which fits tightly into a SANS highpressure cell that has been used extensively for previous neutron scattering experiments with supercritical $\mathrm{CO}_{2}$-polymer mixtures and water $/ \mathrm{CO}_{2}$ microemulsions [13]. Because the influence of the aerogel on the phase diagram of confined 
$\mathrm{CO}_{2}$ is not known definitely, temperature scans in the range $80^{\circ} \geqslant T \geqslant 25^{\circ} \mathrm{C}$ were performed at five different constant average densities of $\mathrm{CO}_{2}$ (Matheson Gas Products, Inc, SFC purity 99.99\%): $\quad \rho_{\mathrm{CO}_{2}}=0.3, \quad 0.4, \quad 0.468, \quad 0.55$, and $0.65 \mathrm{~g} / \mathrm{cm}^{3}$-i.e., within $\pm 35 \%$ deviation from the critical density of the bulk $\mathrm{CO}_{2}, \rho_{C}=0.468 \mathrm{~g} / \mathrm{cm}^{3}$. The density steps of $0 \%, \pm 17 \%$, and $\pm 35 \%$ of $\rho_{C}$ were chosen based on experimental data on the phase diagrams of ${ }^{4} \mathrm{He}$ and Ne confined in aerogels, which reported a $(17 \%-20 \%)$ and $(\sim 0 \%)$ shift of the critical density of the confined fluids [14]. Two other average densities were selected in order to explore the behavior of $\xi$ at conditions corresponding to the maximal ( $\rho$ $\left.=0.3 \mathrm{~g} / \mathrm{cm}^{3}\right)$ and minimal $\left(\rho=0.65 \mathrm{~g} / \mathrm{cm}^{3}\right)$ absorption regimes, respectively [15]. The pressure was measured using a precision digital pressure indicator (Sensotec, model AG100) and the temperature of the cell was controlled to better than $\pm 0.2{ }^{\circ} \mathrm{C}$.

SANS experiments were performed on the KWS-2 SANS facility at the FRJ reactor in Julich, Germany. The neutron wavelength was $\lambda=6.3 \AA(\Delta \lambda / \lambda=0.10 \%)$. Three sampledetector distances of $14 \mathrm{~m}, 4 \mathrm{~m}$, and $1.4 \mathrm{~m}$ were used for measuring scattering from empty aerogel to give an overall range of momentum transfer $0.003<Q$ $=4 \pi \lambda^{-1} \sin \theta<0.2 \AA^{-1}$, where $2 \theta$ is the scattering angle. Measurements of empty aerogel were repeated after each temperature scan and revealed no changes in the aerogel structure after exposure to $\mathrm{CO}_{2}$. Scattering from bulk and confined $\mathrm{CO}_{2}$ was measured in the appropriate $Q$ range $\left(0.007<Q<0.07 \AA^{-1}\right)$. The SANS data sets were corrected for instrumental backgrounds and normalized to an absolute $( \pm 5 \%)$ differential cross section per unit sample volume $\left[I(Q)\right.$ in units of $\left.\mathrm{cm}^{-1}\right]$ by means of precalibrated secondary standards [16].

The intensity of scattering from empty aerogel is shown in the inset of Fig. 1. We find that $I(Q)$ is best described by the function [17]

$$
I(Q)=\frac{I(0)}{\left[1+Q^{2} \xi_{G}^{2}\right]^{\left(D_{f}-1\right) / 2}} \frac{\sin \left[\left(D_{f}-1\right) \tan ^{-1}\left(Q \xi_{G}\right)\right]}{\left(D_{f}-1\right) Q \xi_{G}},
$$

where $\xi_{G}$ is the gel correlation length, which represents the large-length-scale limit of the fractal correlations, $D_{f}$ is the fractal dimension, and $I(0)$ is the extrapolated intensity of scattering at $Q=0$. The solid line is the result of fitting Eq. (1) to the data, which provides an excellent fit in the $Q$ range up to $\sim 0.1 \AA^{-1}$, after which a crossover to the Porod law is observed. The values of fit parameters are $I(0)$ $=57.3 \pm 0.5 \mathrm{~cm}^{-1}, \xi_{G}=85 \pm 9 \AA$, and $D_{f}=2.5 \pm 0.1$, the last two of which are in good agreement with $\xi_{G}$ and $D_{f}$ of basecatalyzed aerogels of similar density [18].

Several representative scattering curves from bulk and confined $\mathrm{CO}_{2}$ as a function of temperature in the one-phase region are shown in Fig. 1. The values of $\xi_{\text {bulk }}$ for bulk $\mathrm{CO}_{2}$ as well as $I(0)$ proportional to the fluid compressibility were obtained by fitting $I(Q)$ to the Ornstein-Zernike formula

$$
I(Q)=\frac{I(0)}{\left(1+Q^{2} \xi^{2}\right)} .
$$

As shown in Fig. $1, I(Q)$ from the bulk fluid changes significantly as $T_{C}$ is approached. Thus, $I(0) \cong 0.08 \mathrm{~cm}^{-1}$ at $T$

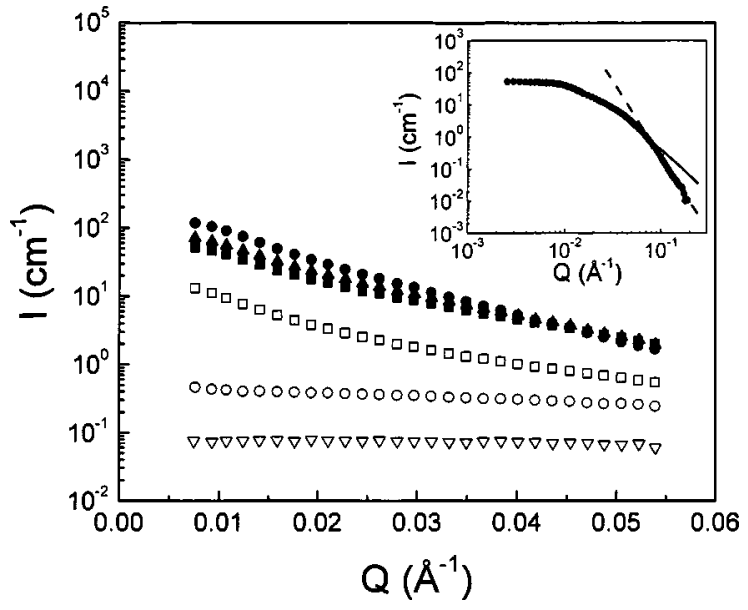

FIG. 1. Absolute neutron scattering cross section per unit volume measured from bulk (open symbols) and confined $\mathrm{CO}_{2}$ (solid symbols) in the homogeneous region. $(\nabla, \boldsymbol{\square}) T=80{ }^{\circ} \mathrm{C},(\bigcirc, \boldsymbol{\Delta}) T$ $=40{ }^{\circ} \mathrm{C}$, and $(\square, 0) T=31.4{ }^{\circ} \mathrm{C}$. The average density of $\mathrm{CO}_{2}$ is $\rho_{C}=0.468 \mathrm{~g} / \mathrm{cm}^{3}$. The inset shows scattering from empty aerogel at ambient conditions. The solid line is described by Eq. (1), whereas the dashed line corresponds to the Porod law $I \sim Q^{-4}$.

$=80{ }^{\circ} \mathrm{C}$ increases by more than two orders of magnitude up to $I(0) \cong 25 \mathrm{~cm}^{-1}$ and $\xi_{\text {bulk }} \cong 6.4 \AA\left(T=80^{\circ} \mathrm{C}\right)$ increases up to $\xi_{\text {bulk }} \cong 123 \AA$ at $T-T_{C}=0.4^{\circ}\left[T_{C}=31 \pm 0.2{ }^{\circ} \mathrm{C}\right.$ of bulk $\mathrm{CO}_{2}$ was estimated from extrapolation of the $I(0, T)^{-1}$ to zero and agrees well with generally accepted value $31.06{ }^{\circ} \mathrm{C}$ [15]]. The intensity of scattering from $\mathrm{CO}_{2}$-saturated aerogel at $T$ $=80{ }^{\circ} \mathrm{C} \gg T_{C}$, is generally lower than that from empty aerogel due to a lower neutron contrast between $\mathrm{SiO}_{2}$ and compressed $\mathrm{CO}_{2}$. However, the $Q$ dependence of the scattering is virtually identical to that of empty aerogel. As the temperature is decreased toward the critical point, the intensity increases more rapidly at low $Q$; however, the relative increase in $I(0)$ is moderate compared to that of the bulk fluid (about a factor of 2 versus two orders of magnitude over the same range of $T-T_{C}$, Fig. 1). The variation of $\xi_{\text {bulk }}$ as a function of $T$ and the reduced temperature $\tau=\left(T-T_{C}\right) / T_{C}$ is shown in Figs. 2 and 3, respectively. As mentioned above, $\xi_{\text {bulk }}$ tends to diverge as $T \Rightarrow T_{C}$ and decreases rapidly below $T_{C}$ at which point the formation of the meniscus is observed. On the log-log scale, the slope of $\xi_{\text {bulk }}(\tau)$ gives the critical index of the bulk correlation length $\nu_{\text {bulk }}=0.61 \pm 0.02$ (Fig. 3), which agrees within experimental error with the Ising model exponent $\nu=0.630 \pm 0.001$ [19] as well as with results of previous studies [20].

The scattering results for confined $\mathrm{CO}_{2}$ can be interpreted quantitatively using an equation similar to that utilized previously to interpret SANS results on the influence of confinement on the liquid-liquid critical phenomena in partially miscible liquid solutions [7]:

$$
I(Q)=\frac{I_{L O R}}{\left(1+Q^{2} \xi^{2}\right)}+\left[\frac{I_{L S Q}}{\left(1+Q^{2} \xi^{2}\right)}\right]^{2} I_{\mathrm{bgr}}(Q)+C .
$$

In the one-phase region, it corresponds to the sum of a Lorentzian $\left(1+Q^{2} \xi^{2}\right)^{-1}$ and Lorentzian-squared term (1 


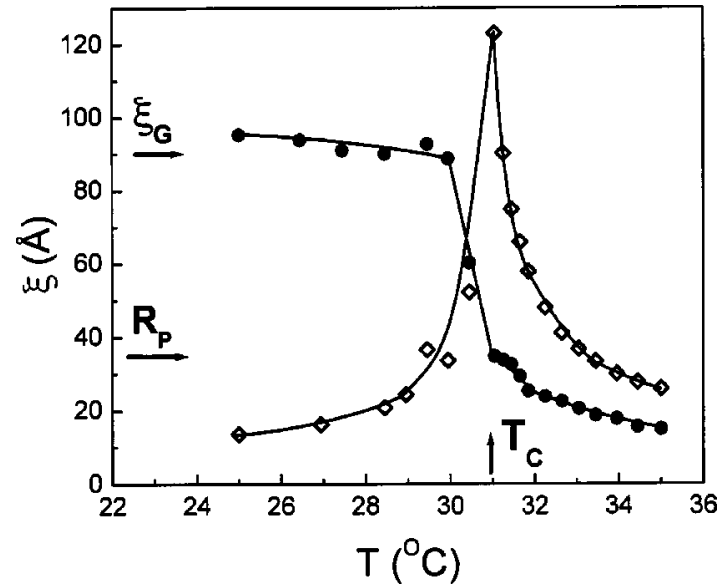

FIG. 2. Correlation length of the density fluctuations of $\mathrm{CO}_{2}$ in bulk $(\diamond)$ and under confinement $(\bullet)$.

$\left.+Q^{2} \xi^{2}\right)^{-2}$. The angle-independent background (C $\sim 0.5 \mathrm{~cm}^{-1}$ ) arises mainly from $\mathrm{H}^{1}$ atoms in the methoxy groups on the aerogel surface. The Lorentzian term is identical to Eq. (2) and describes the genuine scattering from critical fluctuations inside pores, whereas the Lorentziansquared term arises from the response of the order parameter (fluid density) to the "field" induced by aerogel and is related to the critical absorption [21]. The scattering from aerogel itself is taken into account in Eq. (3) via the "background" term $I_{\text {bgr }}(Q)$, which represents scattering from $\mathrm{CO}_{2}$-saturated aerogel at $T=80{ }^{\circ} \mathrm{C} \gg T_{C}$, when contribution of the fluctuations of the order parameter is negligible (see Fig. 1). Equation (3) is similar to expressions used to fit neutron scattering data for diluted antiferromagnets in an external magnetic field [22] as well as to equations predicted for liquid solutions in porous networks based on extensions of the RFIM $[2,3]$.

We have found that in the one-phase region $T>31{ }^{\circ} \mathrm{C}$, $I(Q)$ is best described ( $\chi^{2}$ below 2 ) by Eq. (3) with the same value of $\xi$ in both Lorentzian and Lorentzian-squared terms,

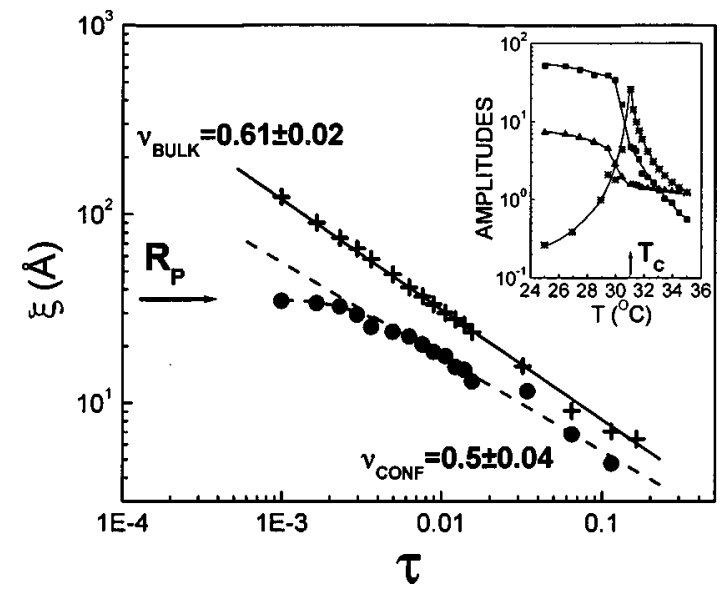

FIG. 3. $\xi_{\text {bulk }}(+)$ and $\xi_{\text {conf }}(\bigcirc)$ vs reduced temperature $\tau=(T$ $\left.-T_{C}\right) / T_{C}\left(T_{C}=31{ }^{\circ} \mathrm{C}\right)$. The inset shows the temperature variation of amplitudes in Eqs. (2) and (3). Bulk $\mathrm{CO}_{2}(*)\left[I(0), \mathrm{cm}^{-1}\right]$. Confined $\mathrm{CO}_{2}(\mathbf{\Delta})\left[I_{L O R}, \mathrm{~cm}^{-1}\right],(\square)\left[I_{L S Q}\right]$.

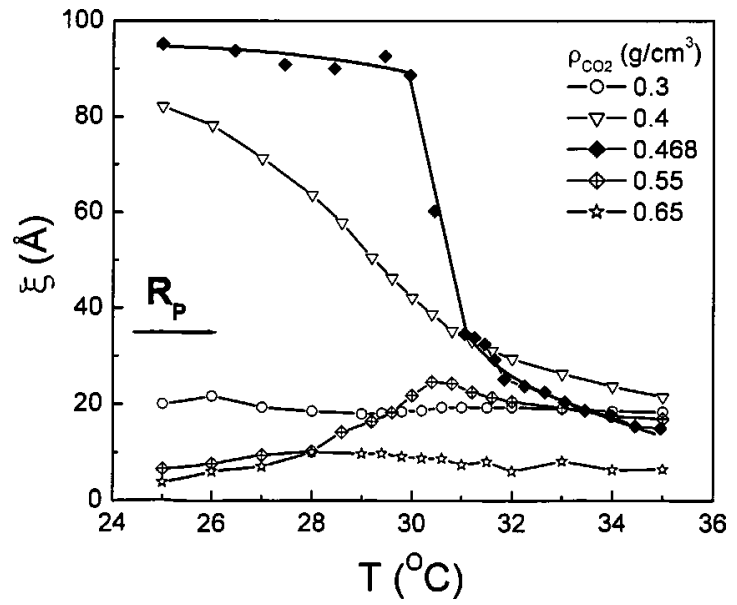

FIG. 4. Temperature variation of $\xi_{\text {conf }}$ at different average densities of $\mathrm{CO}_{2}$.

as theoretically predicted [23]. This is in agreement with results obtained for liquid solutions in rigid porous matrices of Vycor glass and silica gels, but is in contrast to the case of the solutions in soft polymer gels, where the fitting procedure calls for very different values for $\xi$ in the two terms [24]. The temperature variation of the correlation length of confined $\mathrm{CO}_{2} \quad\left(\xi_{\text {conf }}\right)$ at average density $\rho=\rho_{C \text {,bulk }}$ $=0.468 \mathrm{~g} / \mathrm{cm}^{3}$ is shown in Figs. 2 and 3. At $T>T_{C}=31{ }^{\circ} \mathrm{C}$, in the one-phase region, $\xi_{\text {conf }}$ is systematically smaller than $\xi_{\text {bulk }}$ and the difference between the two parameters increases as $T \Rightarrow T_{C}$. These trends arise because $\xi_{\text {bulk }}$ diverges and $\xi_{\text {conf }}$ saturates at the value corresponding to the pore radius $R_{P}$ $\sim 30-35 \AA$. The log-log slope of $\xi_{\text {conf }}(\tau)$ gives $\nu_{\text {conf }}$ $=0.5 \pm 0.04$ in the range $\tau \geqslant 6 \times 10^{-3}$, which is close to the mean field value of $\nu=0.5$ [15]. At $\tau \Rightarrow 0, \xi_{\text {conf }}$ tends to round off at $\xi_{\text {conf }} \sim R_{P}$. Below $T=31{ }^{\circ} \mathrm{C}$, both $\xi_{\text {conf }}$ and the amplitudes $I_{L O R}$ and $I_{L S Q}$ in Eq. (3) show a discontinuous change in the fit parameters and $\xi_{\text {conf }}$ tends to saturate at a value roughly corresponding the correlation length of aerogel, $\xi_{G}$ $\sim 90 \AA$ A. At this point aerogel becomes strongly opaque, but exhibits no evidence of macroscopic phase separation (i.e., formation of meniscus). We speculate that this results from the formation of "frozen" metastable structural domains which mimic the correlated disordered introduced by the aerogel matrix. The intensity of scattering increases by two orders of magnitude and does not change with time at fixed temperature, which confirms the stability of the domains below $T_{C}$. These results are in qualitative agreement with the light transmission measurements below $T_{C}$ of $\mathrm{N}_{2}$ confined in an aerogel [25], which revealed critical light opalescence and the invariable values of the transmitted light intensity below $T_{C}$ at fixed temperature, as well as the absence of macroscopic phase separation.

We interpret the lack of critical divergences of $\xi$ and $I(0)$ as the result of the competition between the genuine critical fluctuations in the "core" of aerogel pores and critical absorption of $\mathrm{CO}_{2}$ molecules on the silica strands of the aerogel, as both processes are characterized by a similar correlation length. Because the average density of $\mathrm{CO}_{2}$ in the aerogel remains globally conserved and some fraction of molecules becomes adsorbed and ceases to participate in 
critical fluctuations, the local density of the remaining fluid may become somewhat depleted in $\mathrm{CO}_{2}$ [26]. To assess whether this effect can shift the critical density of the confined fluid, we have measured the variation of the correlation length at average densities of $\mathrm{CO}_{2}$ in the range of $\rho_{C \text {,bulk }} \pm 35 \%$. The results shown in Fig. 4 demonstrate that $\xi$ always remains $<R_{P}$ in the homogeneous domain and thus the general trend of the density fluctuation suppression is retained at all thermodynamic conditions studied in this work.

In conclusion, results have been presented on SANS measurements used to assess the influence of dilute disorder on the fluid density fluctuations near the liquid-gas critical point of $\mathrm{CO}_{2}$. We found no evidence of RFIM behavior in the range of scattering vectors, reduced temperatures, and average densities of $\mathrm{CO}_{2}$ covered in our experiments. The dilute quenched disorder induced by the host matrix works to suppress density fluctuations in the critical region due to a critical depletion mechanism and the correlation length of the order parameter does not exceed the characteristic pore size in the homogeneous region. Our results provide evidence for individual fluids in aerogels to fall in the category of phase separation under confinement with critical absorption effects, in qualitative agreement with the single-pore model. Experiments with various types of porous hosts and different fluids are currently in progress in order to elucidate the influence of molecule-surface interactions and pore topology on the liquid-gas critical phenomena in confined fluids.

We thank M. E. Fisher and S. K. Sinha for a critical reading of the manuscript and helpful remarks and acknowledge helpful conversations with F. Aliev, M. Droege, E. Eisenriegler, and K. E. Gubbins. This research was sponsored by the Laboratory Directed Research and Development Program of Oak Ridge National Laboratory, managed by UTBattelle, LLC, for the U.S. Department of Energy under Contract No. DE-AC05-00OR2272.
[1] For a recent review see L. D. Gelb, K. E. Gubbins, R. Radhakrishnan, and M. Sliwinska-Bartkowiak, Rep. Prog. Phys. 62, 1573 (1999); K. Binder and E. Luijten, Phys. Rep. 344, 179 (2001).

[2] D. Andelman and J. F. Joanny, in Scaling Phenomena in Disordered Systems, edited by R. Pynn and A. Skjeltorp (Plenum, New York, 1985).

[3] F. Brochard and P. G. de Gennes, J. Phys. (France) Lett. 44, 785 (1983); P. G. de Gennes, J. Phys. Chem. 88, 6469 (1984).

[4] A. J. Liu et al., Phys. Rev. Lett. 65, 1897 (1990).

[5] J. V. Maher et al., Phys. Rev. Lett. 53, 60 (1984); S. B. Dierker and P. Wiltzius, ibid. 66, 1185 (1991); Z. Zhuang, A. G. Casielles, and D. S. Cannell, ibid. 77, 2969 (1996); F. Formizano and J. Teixera, Eur. Phys. J. E 1, 1 (2000).

[6] L. Monette, A. J. Liu, and G. S. Grest, Phys. Rev. A 46, 7664 (1992).

[7] B. J. Frisken et al., Phys. Rev. E 51, 5866 (1995).

[8] K. Q. Xia and J. V. Maher, Phys. Rev. A 37, 3626 (1988); B. J. Frisken and D. S. Cannell, Phys. Rev. Lett. 69, 632 (1994); M. Y. Lin et al., ibid. 72, 2207 (1994).

[9] S. K. Sinha, in Methods in the Physics of Porous Media, edited by P. Z. Wong (Academic Press, London, 1999).

[10] Aerogels, edited by J. Fricke (Springer-Verlag, Berlin, 1988).

[11] S. Lacelle et al., Phys. Rev. Lett. 74, 5228 (1995); L. Tremblay, S. M. Socol, and S. Lacelle, Phys. Rev. E 61, 656 (2000).

[12] BET data supplied by the manufacturer. See also K. Tajiri, K. Igarashi, and T. Nishio, J. Non-Cryst. Solids 186, 83 (1995); E. Anglaret et al., ibid. 186, 186 (1995).

[13] Y. B. Melnichenko et al., Macromolecules 32, 5344 (1999); S. Senapati et al., Langmuir 18, 7371 (2002).

[14] A. P. Y. Wong and M. H. W. Chan, Phys. Rev. Lett. 65, 2567
(1990); H. W. Tan and J. R. Beamish, Physica B 284-288, 389 (2000); T. Herman and J. R. Beamish, J. Low Temp. Phys. 126, 661 (2002).

[15] O. Di Giovanni et al., Langmuir 17, 4316 (2001).

[16] G. D. Wignall and F. S. Bates, J. Appl. Crystallogr. 20, 28 (1986).

[17] S. K. Sinha, T. Freltoft, and J. Kjems, in Kinetics of Aggregation and Gelation, edited by F. Family and D. P. Landau (North-Holland, Amsterdam, 1984); T. Freltoft, J. K. Kjems, and S. K. Sinha, Phys. Rev. B 33, 269 (1986).

[18] R. Vacher et al., Phys. Rev. B 37, 6500 (1988).

[19] J. C. LeGuillou and J. Zinn-Justin, Phys. Rev. B 21, 3976 (1980).

[20] H. L. Swinney and H. Z. Cummings, Phys. Rev. 171, 152 (1968); B. Chu and J. S. Lin, J. Chem. Phys. 53, 4465 (1970).

[21] M. E. Fisher and P. G. de Gennes, Acad. Sci., Paris, C. R. 287, 207 (1978).

[22] See, e.g., Z. Slanic, D. P. Belanger, and J. A. Fernandez-Baca, Phys. Rev. Lett. 82, 426 (1999), and references therein.

[23] D. J. Wallace and R. K. P. Zia, Phys. Rev. Lett. 43, 808 (1979).

[24] S. K. Sinha, J. Huang, and S. K. Satija, in Scaling Phenomena in Disordered Systems (Ref. [2]). See also Maher et al. (Ref. [5]) and Xia and Maher (Ref. [8]).

[25] A. P. Y. Wong et al., Phys. Rev. Lett. 70, 954 (1993).

[26] H. Nakanishi and M. E. Fisher, J. Chem. Phys. 78, 3279 (1983); M. Thommes and G. H. Findenegg, Langmuir 10, 4270 (1994); M. Thommes, G. H. Findenegg, and M. Schoen, ibid. 11, 2137 (1995); M. Schoen, M. Thommes, and G. H. Findenegg, J. Chem. Phys. 107, 3262 (1997). 